\title{
INVESTIGATION OF BOVINE HERPESVIRUS TYPE -1- (BHV-1) ANTIBODIES IN SYRIAN CATTLE
}

S.Y. AL-BAROODI , A. KURDI , and A. ALOMAR

Dept. of Microbiology, Faculty of Veterinary Medicine, Al-Baath University, Syria Arab Republic.

\section{ABSTRACT}

\section{Received:11/6/2012}

Accepted at:
In order to investigate seroprevalence Bovine Herpesvirus-1- antibodies in dairy cattle farms in Syria , 230 serum samples were collected from cattle. The age, sex and relationship with percentage of infection with Bovine herpesvirus-1- were studied by using Competitive Enzyme Linked Immunosorbant assay (cELISA) and Neutralization test(NT). The total percentage of seropostive cattle was $45.21 \%$ by using cELISA, while the lowest percentage was recorded $36.09 \%$ by using NT . The results indicated the high infection were found in Gub Ramla 60\%,while the lowest percentage was detected in Zerba $30 \%$ by using both tests . Results showed high percentage of acute infection in the age less than 6 months to both sex, while the high percentage of chronic infection recorded in female $(2<-4$ years) .No chronic infection recorded in young both sex calves .High percentage of acute infection detected in Jourin, while high percentage of chronic infection recorded in Gub Ramla .Comparison between the two tests used in this study, Showed that high percentages of infection in cELISA ,while in NT recorded less percentages. The high percentage of infection appear in cattle aborted in $2^{\text {nd }}$ trimester of pregnancy when compared with other ones, high percentage were recorded in cattle which suffer from reproductive disorders. The high percentage of infection with Bovine herpesvirus-1- recorded in animals which suffer from respiratory signs ,while the lowest infection rate recorded in healthy one, without any significant of variance.

Key Words: Bovine herpesvirus-1-Enzyme Linked Immunosorbent Assay-Competitive -serum Neutralization testImmunoglobulin IgM-Immunoglobulin IgG-Acute infection-Chronic infection.

$$
\text { التقصي عن أضداد الفيروس الحلئي البقري النمط ـ اـ في الأبقار في سوريا }
$$

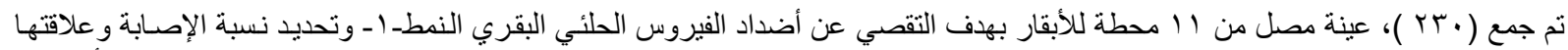

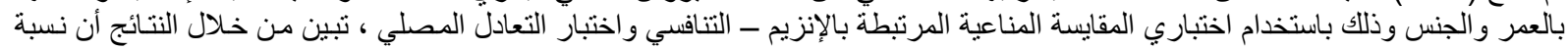

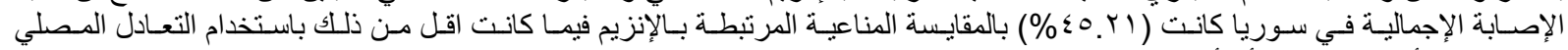

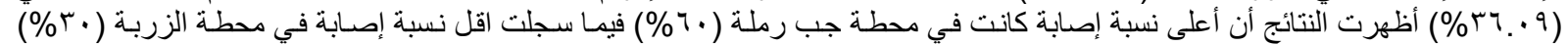

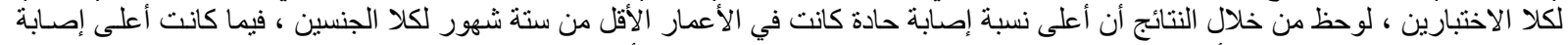

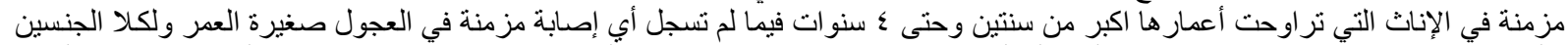

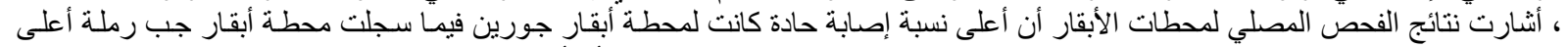

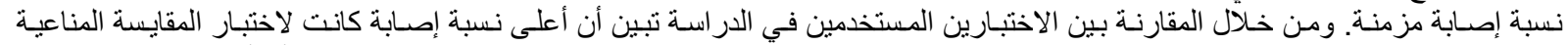

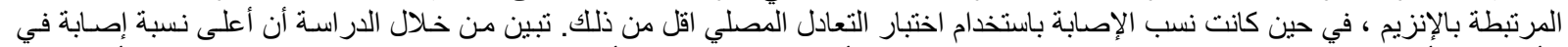

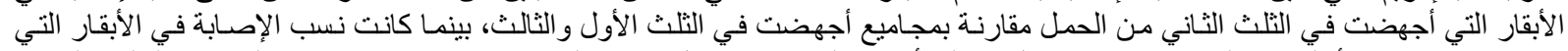

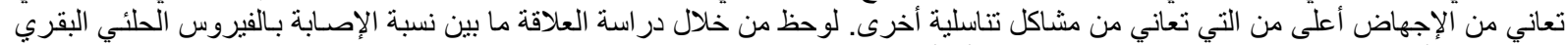

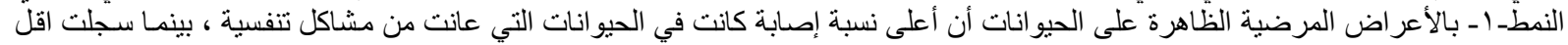

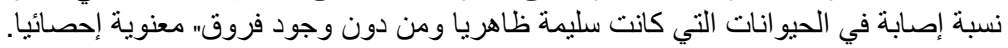

\section{INTRODUCTION}

$$
\text { المقدمـة }
$$

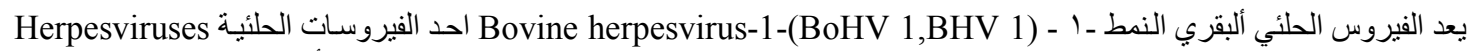

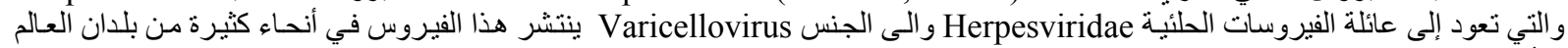

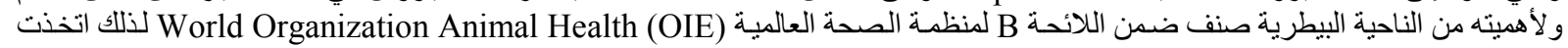

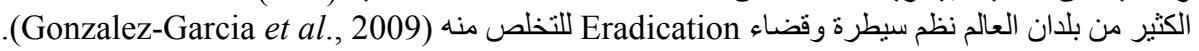




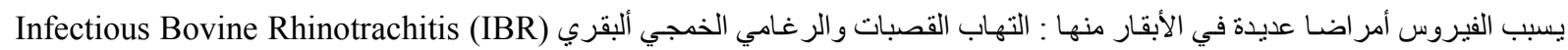

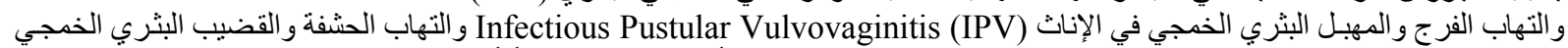

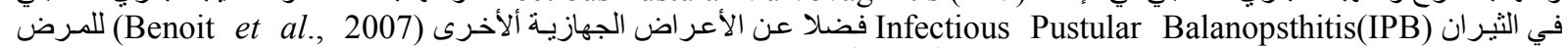

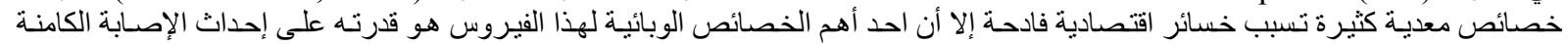

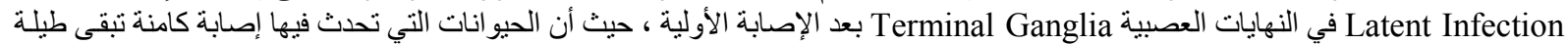

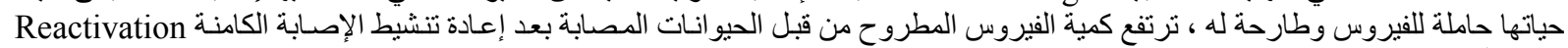

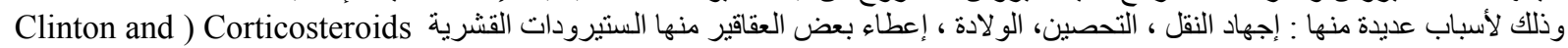

.(Shafiqul, 2008

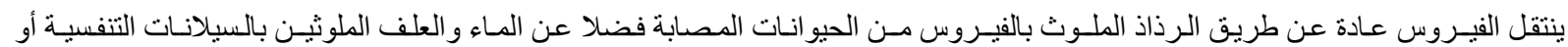

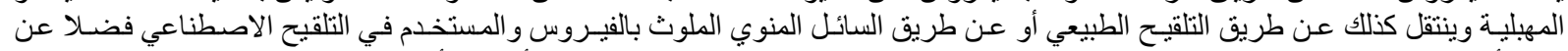

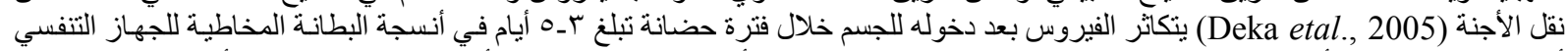

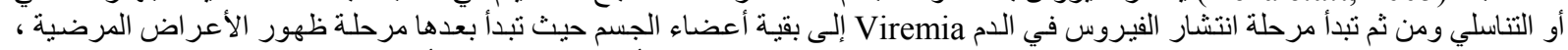

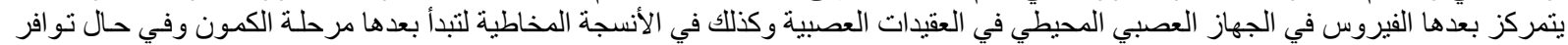

الظروف الملائمة تعاد عملية إعادة تنشيط الإصابة (Ezzi et al., 2011).

و لأهمية الفيروس من الناحية الاقتصادية فقد بدأت الكثبر من الدول نظاما للسيطرة عليه وأخذت تتبع بر امج للتشخيص ومـن ثـم السيطرة واستبعـاد

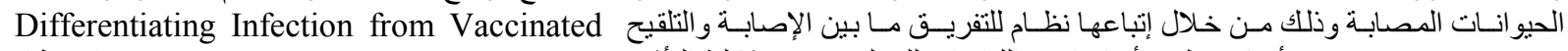
Individual (DIVA)

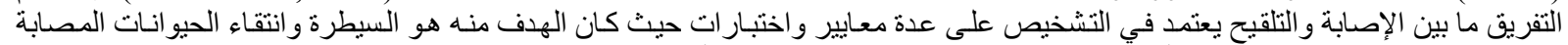

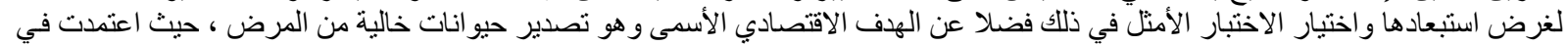

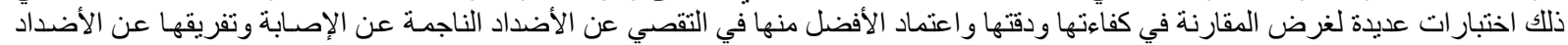

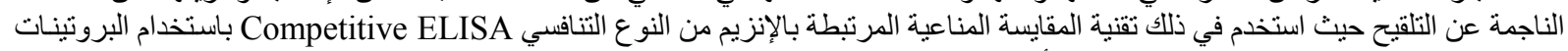

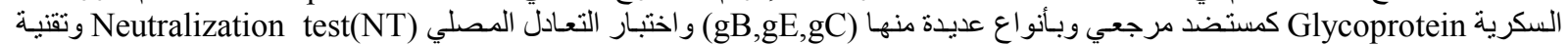

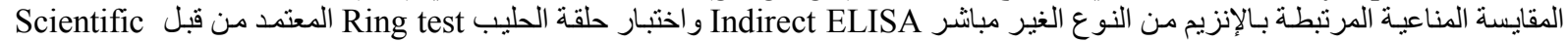
(Julien et al., 2006)Veterinary Committee Group Organized

لذلك تمت هذه الدر اسة نظر العدم وجود در اسة تتناول نسبة الإصـابة في محطات الأبقار في سوريا حيث كانت تهدف إلى الكي: البر هـان عن الأضداد

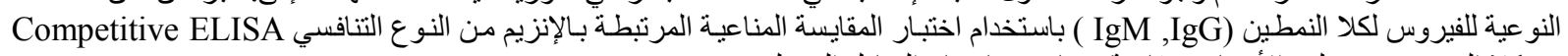
في كلا الجنسين وبمختلف الأعمار ومقارنة حساسيته باختبار التعادل المصلي.

\title{
MATERIALS and METHODS
}

\author{
المواد وطرائق البحث
}

:Animals الحيوانات

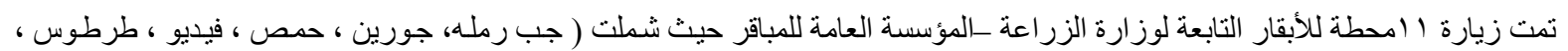

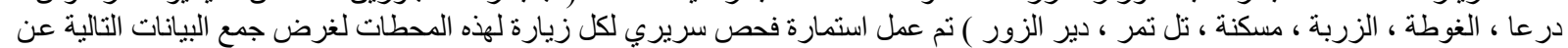

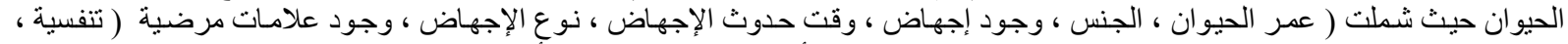

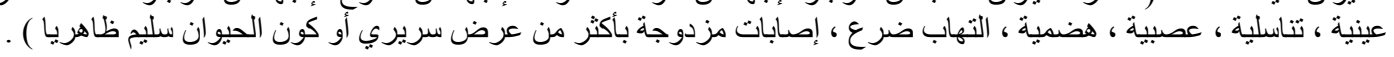

\section{: Sample collection جمع العينات}

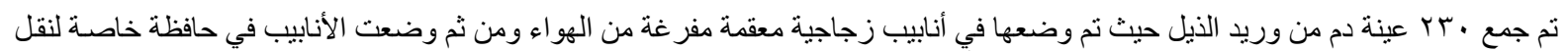

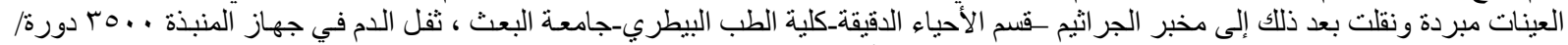

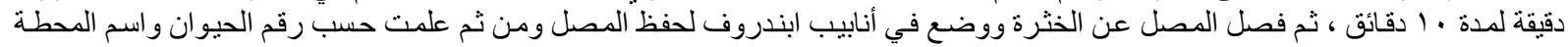
وحفظت عند الدرجة ـ • ب م مه لحين إجر اء الاختبار ات المصلية عليها. (Rai,2005).

بـ الاختبارات المصلية Serological tests

أولا: تقنية المقايسة المناعية المرتبطة بالأنزيم من النوع التنافسي غير المباشر In-Direct Competitive Enzyme Linked Immuno Sorbant Assay (CI-ELISA) Labor Diagnostik Leipzig الألمانية لغرض التقصي عن الغلوبيولينات المناعية بنو عيها وكالاتي : ـ الكواشف والمحاليل الكيميائية والمواد المستخدمة:

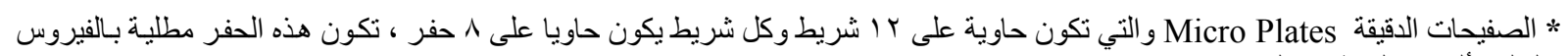

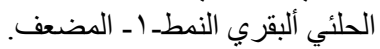

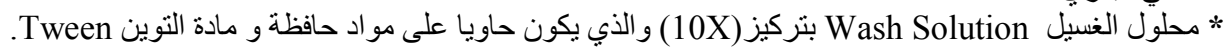

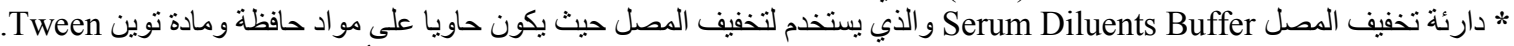

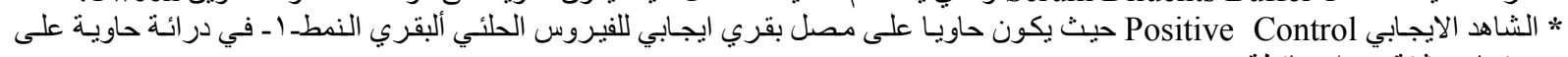
بروتينات مثبتة ومواد حافظة.

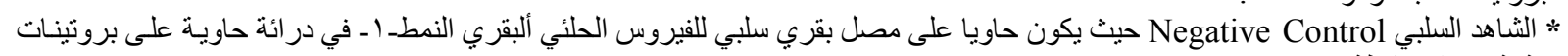
مثبتة ومو اد حافظة. 


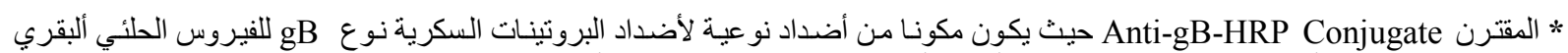

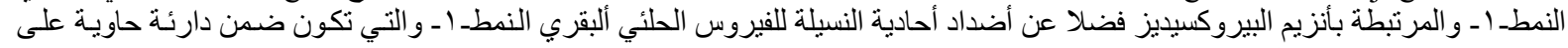
بروتينات مثبتة ومو اد حافظة.

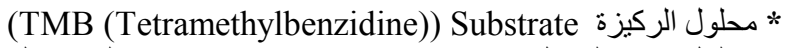

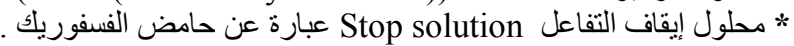

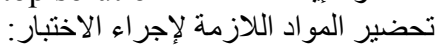

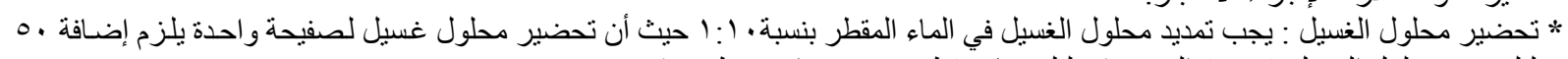

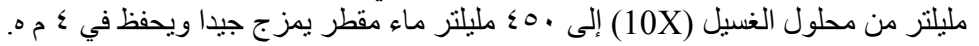

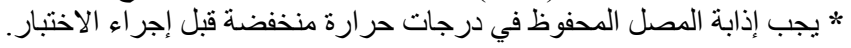

-

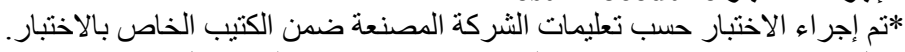

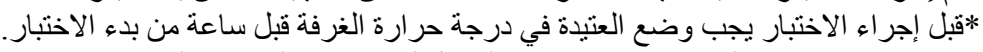

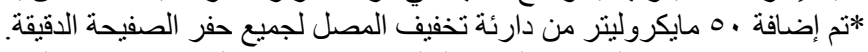

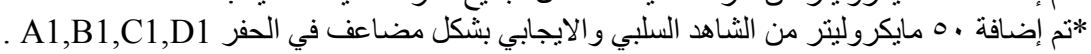

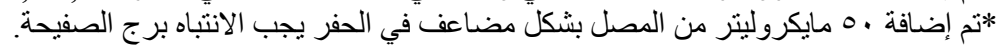

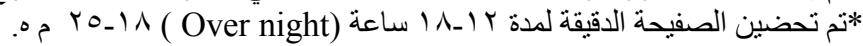

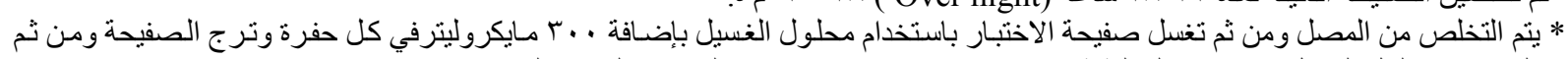

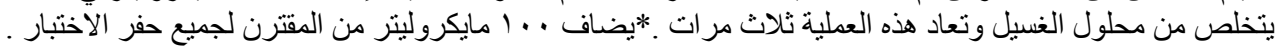

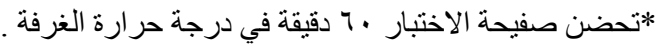

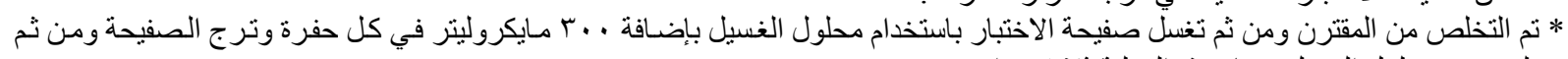

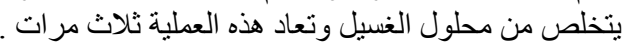

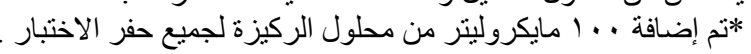

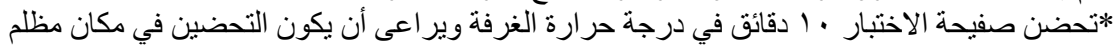

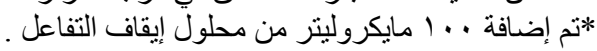

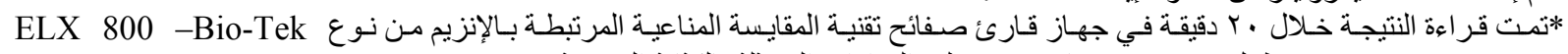

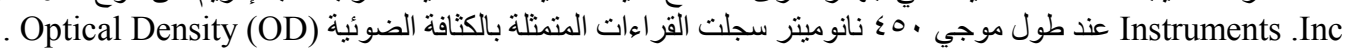

حساب النتائج

* تم حساب متوسط الثاهد السلبي و الايجابي لكل واحد منهما على حده بجمع الكثافة الضوئية لكل واحد منهما وتقسيمها على عدد الحفر لهما.

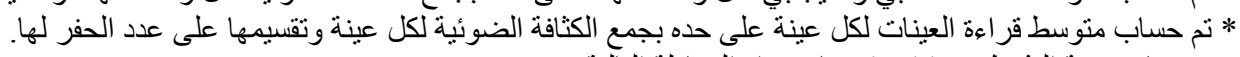

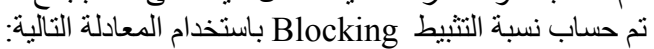

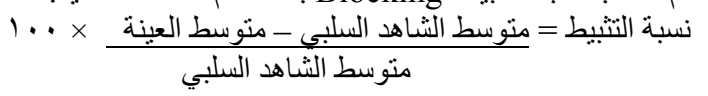
تقييم النتائج :

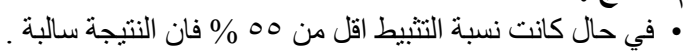

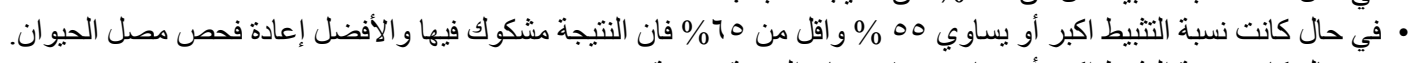

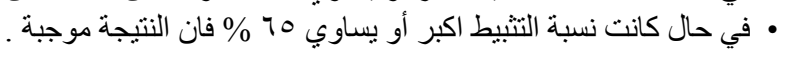

ثانيا: اختبار التعادل المصلي Serum Neutralization(SN)

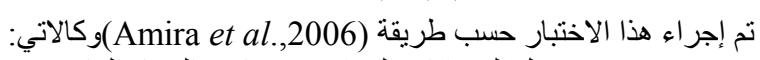

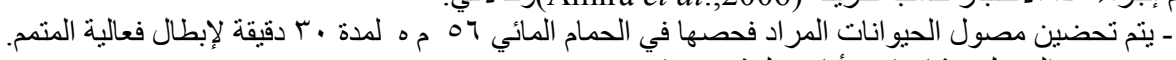

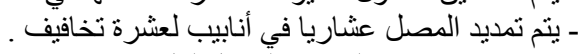

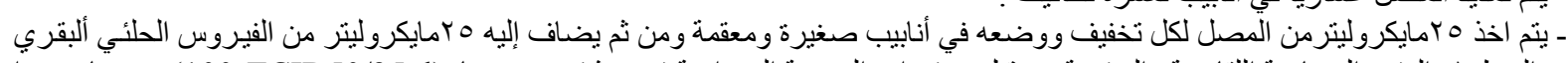

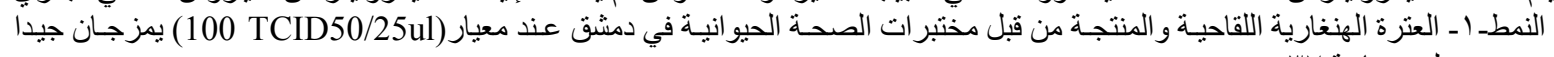

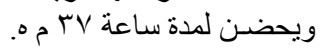

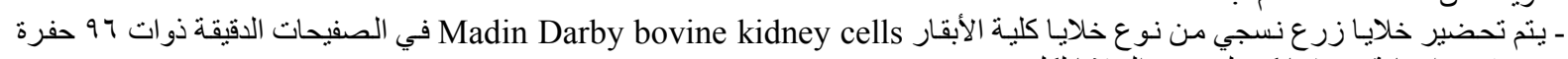

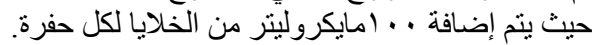

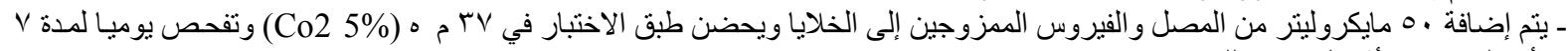

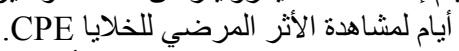

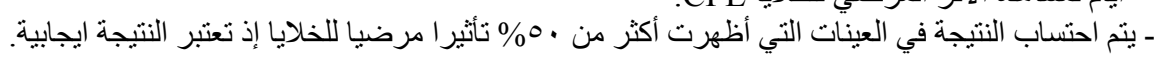

ع - التحليل الإحصائي: تم استخدام اختبار Z ضمن برنامج Sigma state لتحليل النتائج إحصائيا.

\section{RESULTS} النتائج

بينت نتائج الفحص المصلي للفيروس الحلئي البقري النمطــ ا ـ لمحطات الأبقار الحلوب في سوريا باستخدام تقنية المقايسة المناعية المرتبطة بـإلإنزيم

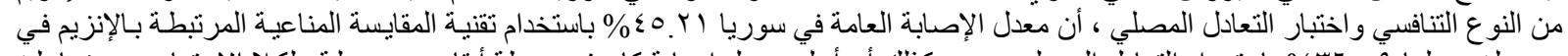

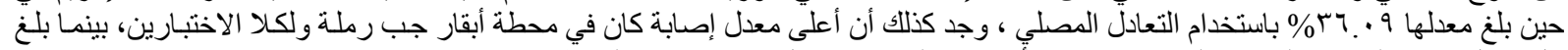

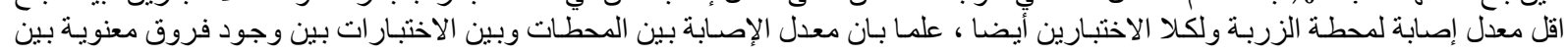

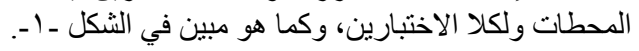




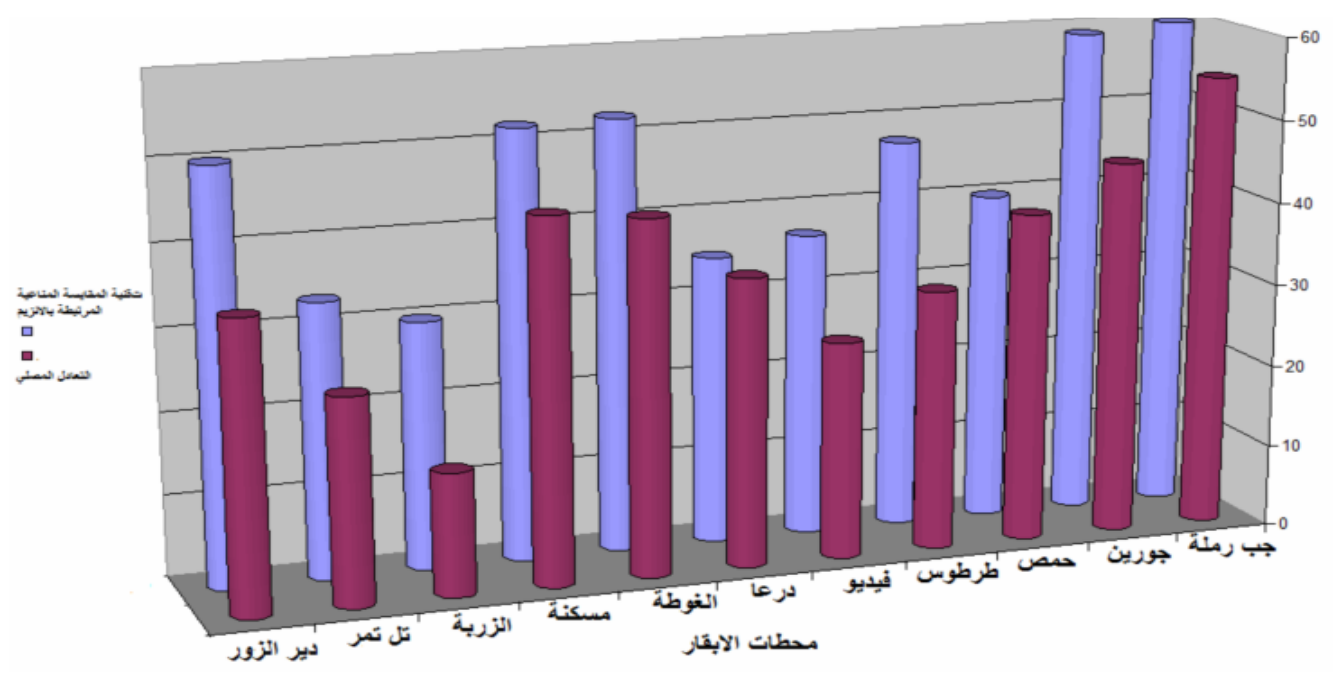

الثكل ا: يبين نسبة الإصابة العامة لمحطات الأبقار في سوريا بالفيروس الحلئي البقري النمطـ اــ باستخدام تقنية المقايسة المناعية المرتبطة

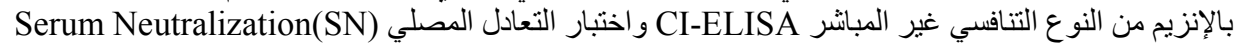

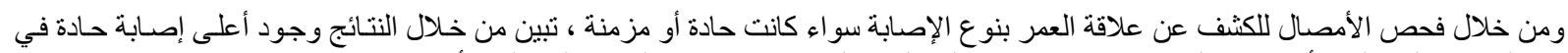

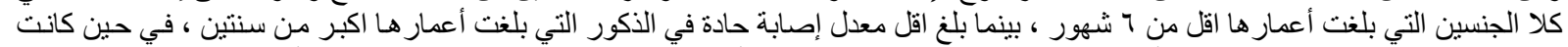

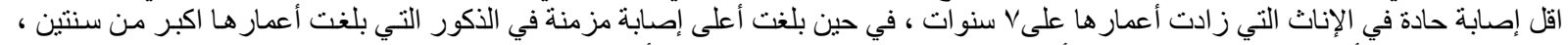

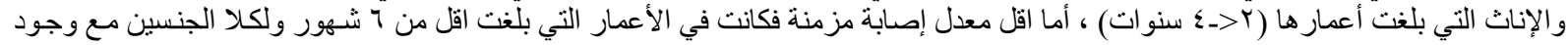

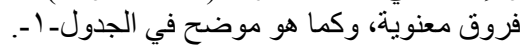
جدول 1: يبين علاقة العمر و الجنس بنسبة الإصابة ونو عها بالفيروس الحلئي البقري النمطـ ا ـ باستخدام تقنيـة المقايسة المناعية المرتبطـة بـالإنزيم من النوع التنافسي غير المباشر

\begin{tabular}{|c|c|c|c|c|c|c|}
\hline 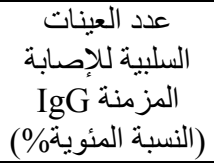 & 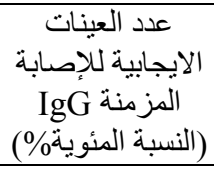 & 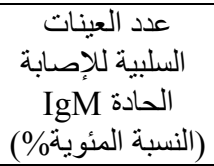 & عدد العينات الايجابية الإبنة الحادة & العينات & العمر & الجنس \\
\hline$(1 \cdots)^{\mu} \cdot$ & $(\cdot) \cdot$ & $(0 r . r \varepsilon) \backslash 7$ & $(\xi 7.77)) \leqslant$ & $r$. & اقل من 7 شهور & \multirow{3}{*}{ الذكور } \\
\hline$(\nu \cdot r \lambda)^{19}$ & $\left(r^{q} .7 r\right)^{\wedge}$ & $(7 r .9 V)) V$ & $\left.\left(r V_{.} \cdot r\right)\right) \cdot$ & TV & 7 شهور - Tسنة & \\
\hline$(\vee 0)^{9}$ & $\left(Y_{0}\right)^{r}$ & $(91.7 v) 11$ & $\left.(\Lambda . r)^{\prime}\right)$ & IT & اكبر من سنتين & \\
\hline$(1 \cdots)^{\mu_{0}}$ & $(\cdot) \cdot$ & $(01 . \leqslant T) 1 \wedge$ & $(\xi \wedge . \Delta \vee) \backslash \vee$ & ro & اقل من 7 شهور & \multirow{6}{*}{ الإناث } \\
\hline$(\wedge \vee .0) Y)$ & $(1 Y .0)^{r}$ & $\left.(0 \leqslant . M)^{\prime}\right) T$ & $(\leqslant 0 . \wedge r) \backslash 1$ & $T \leqslant$ & T شهور - سنة & \\
\hline$\left.(7 \varepsilon .11)^{\prime}\right)_{0}$ & $(r 0 . \wedge 9) \backslash \leqslant$ & $\left(V^{q} . \leqslant q\right)^{r I}$ & $(Y \cdot .01)^{\wedge}$ & $r q$ & r-> & \\
\hline$(\vee \wedge .90)^{\mu} \cdot$ & $\left(Y^{\prime} . .0\right)^{\lambda}$ & $(9 \leqslant . \vee \varepsilon)^{r q}$ & $\left(0 . Y^{\top}\right)^{r}$ & ri & سنوات V_> & \\
\hline$(\wedge \cdot)^{r} \cdot$ & $(Y \cdot)^{0}$ & $(1 \cdots)^{Y_{0}}$ & $(\cdot) \cdot$ & To & اكبر من V v & \\
\hline$(\wedge r.) \wedge) / \wedge q$ & $(I \vee . \wedge r) \leqslant 1$ & $(V Y . T)(T V$ & $(r V . r q) 7 r$ & $r \mu$. & المجموع & \\
\hline
\end{tabular}

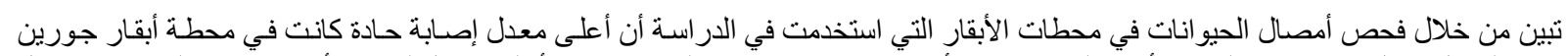

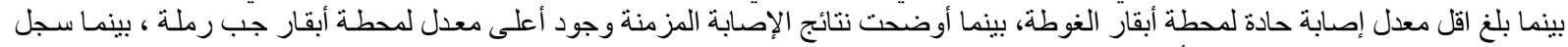

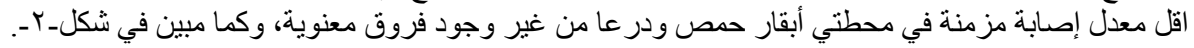




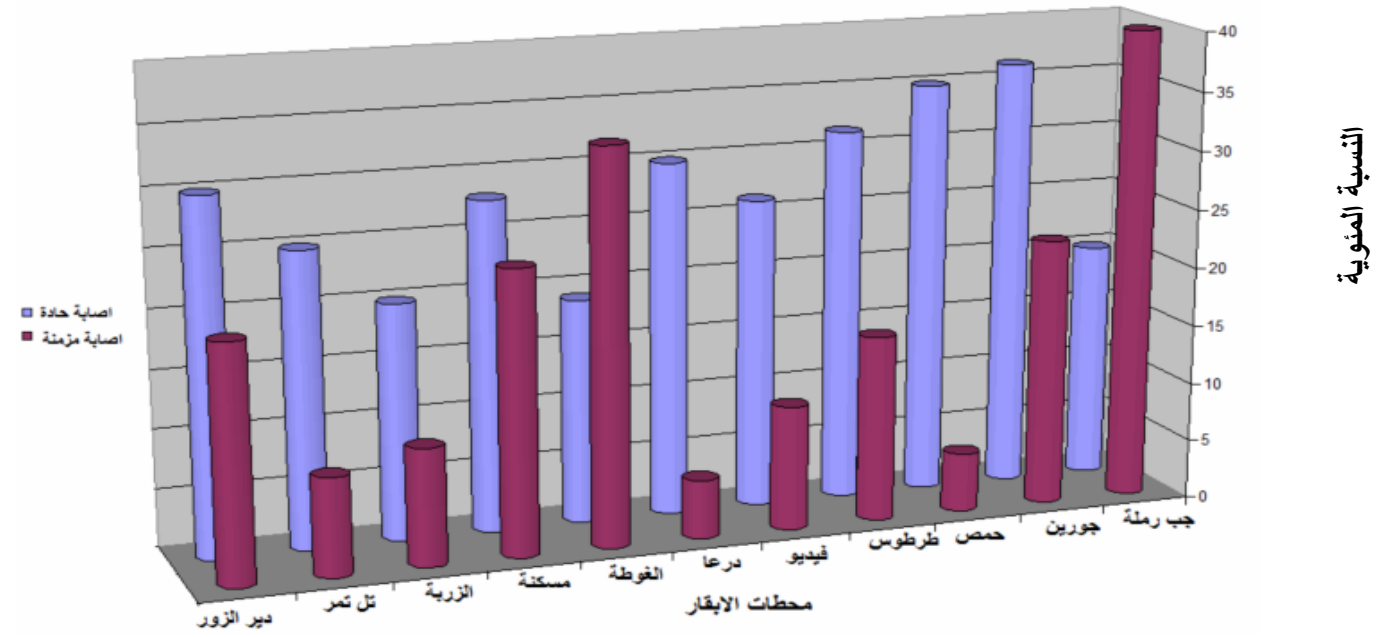

شكل ب : يبين نسبة الإصابة بالفيروس الحلئي البقري النمطـ اـ ـ بنو عيها في محطات الأبقار في سوريا

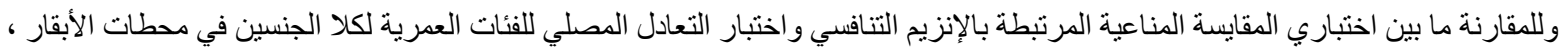

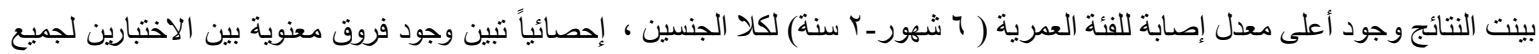

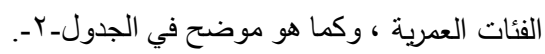

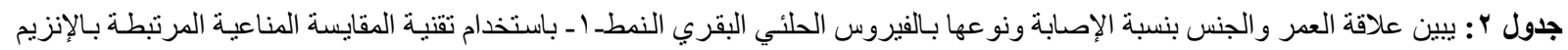
من النوع التنافسي غير المبانشر واختبار التعادل المصلي

\begin{tabular}{|c|c|c|c|c|c|c|}
\hline لاختبار التعادل المصنات السلبية & 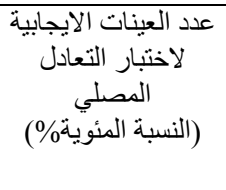 & 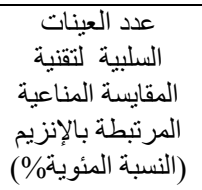 & 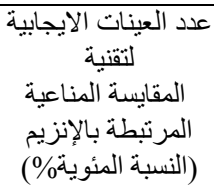 & العينات & العمر & الجنس \\
\hline$(T 7 . T V)^{Y}$. & . & $($ or.rs) 17 & $(\leqslant 7.77)) \leqslant$ & $r \cdot$ & اقل من 7 شهور & \\
\hline$(\varepsilon \varepsilon . \varepsilon \varepsilon) \backslash Y$ & $(00.07)^{10}$ & 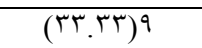 & $(77.7 V)^{\prime \wedge}$ & TV & 7 شهور Tr سنة & \\
\hline$\left(V_{0}\right)^{q}$ & $\left(r^{0}\right)^{r}$ & $(77.7 \vee)^{\wedge}$ & $(\Gamma \mu . \mu T) \varepsilon$ & Tr & اكبر من سنتين & \\
\hline$(\tau \cdot)^{r}$ & $(\xi \cdot) ! \xi$ & $(01 . \varepsilon r) \backslash 1$ & $(\varepsilon \wedge . \diamond \vee) \backslash V$ & ro & اقل من 1 شهور & \\
\hline$(0 \leq) V.) \mid r$ & $(\Sigma 0 . \wedge r) \backslash 1$ & $(\varepsilon) . T V)^{\prime}$. & $(0 \wedge, r T) \mid \leq$ & $T \varepsilon$ & 7 شهور Y سنة & الإناث \\
\hline$(01, Y \wedge)^{Y}$. & $(\varepsilon \wedge . \vee Y)^{\prime} q$ & $(\varepsilon r .0 q) \backslash V$ & $(0 T . \S)$ YY & rq & r> & \\
\hline$(11.01)^{\mu 1}$ & $(1 \wedge . \varepsilon Y)^{\vee}$ & $(\vee \Gamma . \top \Lambda) Y \Lambda$ & $\left(Y^{\prime}, Y^{\prime}\right){ }^{\prime}$ & rA & 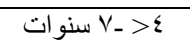 & \\
\hline$\left.(\wedge \varepsilon)^{Y}\right)$ & $(17)^{\varepsilon}$ & $(\wedge \cdot)^{r} \cdot$ & $(r \cdot)^{\circ}$ & ro & اكبر من V سنو ات & \\
\hline$(7 r .91)) \leqslant V$ & $(r 7 . \cdot 9)^{\wedge r}$ & $(0 \leqslant . \vee 9) \backslash Y$ T & $\left.\left(\varepsilon 0, Y^{\prime}\right)\right) \cdot \varepsilon$ & rT. & المجموع & \\
\hline
\end{tabular}

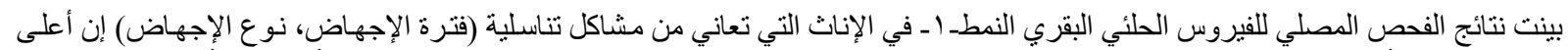

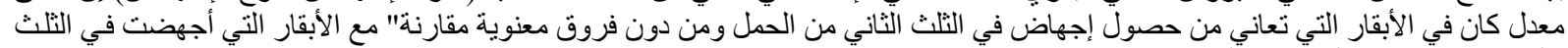

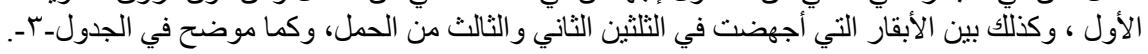

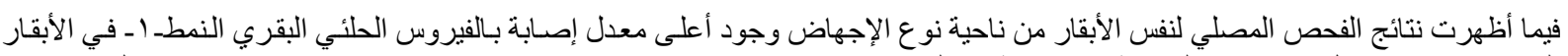

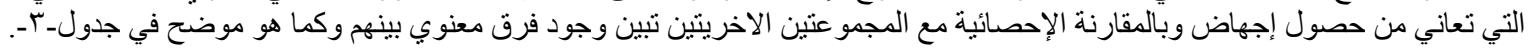


جدول r: يبين علاقة نسبة الإصابة بالفيروس الحئي البقري النمطــ ا ـ بفترة الإجهاض ونو عه

\begin{tabular}{|c|c|c|c|c|}
\hline 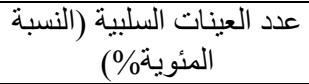 & $\begin{array}{c}\text { عدد العينات الايجابية (النسبة } \\
\text { (المئوية\%) }\end{array}$ & العينات & & \\
\hline$\left(0 V_{.10}\right)^{r}$. & $(\leqslant r . \wedge 0) 10$ & ro & الثلث الأول & \multirow{3}{*}{ الإجهاض فترة } \\
\hline$(0 Y, r q) Y Y$ & $\left(\sum \vee . T\right) Y$. & $\sum r$ & الثلث الثاني & \\
\hline$(\wedge \cdot)^{r} \cdot$ & $(Y \cdot)^{0}$ & TO & الثلث الثالث & \\
\hline$(r 0.07) 17$ & $(7 \leq . \leq \varepsilon)^{Y q}$ & $\leqslant 0$ & إجهاض & \multirow{3}{*}{ الإجهاض } \\
\hline$(\wedge V .1)^{Y V}$ & $(1 Y .9) \varepsilon$ & T & و لادة عجول ميتة & \\
\hline$(V r . A)^{19}$ & $(r 7.9 Y)^{V}$ & $r 7$ & و لادة مبكرة بعجول ضعيفة & \\
\hline
\end{tabular}

بينت نتائج الفحص المصلي للحيو انات التي تعاني من أعر اضا سريريه متباينة وجود أعلى معدل إصابة في الأبقار التي تعاني من أعر اض اض تنفسية

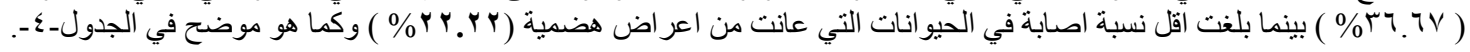

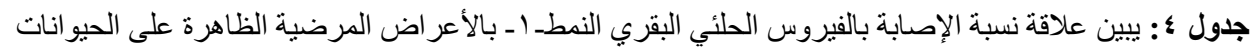

\begin{tabular}{|c|c|c|c|c|}
\hline 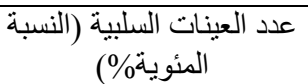 & $\begin{array}{c}\text { عدد العينات الايجابية (النسبة } \\
\text { المئوية\%) }\end{array}$ & العينات & نوع الأعر اض & ت \\
\hline$(r . T V) r Y$ & (Tr.rT) rᄉ & 7. & تنفسية & 1 \\
\hline$(70 . Y V) \leqslant V$ & $(r \leqslant . V T) Y_{0}$ & $V Y$ & تناسلية & $r$ \\
\hline$(\leqslant 0.17) 1 \leqslant$ & $(0 \leq . \wedge \varepsilon) \backslash V$ & $\mu$ & عينية & $r$ \\
\hline$(77.7 V)^{Y}$ & (rT) & $r$ & عصبية & $\varepsilon$ \\
\hline$(\vee \vee . \vee \wedge)^{\vee}$ & $(Y, Y Y)^{\prime}$ & 9 & هضمية & 0 \\
\hline$(0 .)^{\mu}$ & $(0 .)^{r}$ & 7 & التهاب الضرع & 7 \\
\hline$(19.11)^{9}$ & $\left.(\neg \cdot . \wedge V)^{\prime}\right) \leq$ & Tr & مزدوجة & $V$ \\
\hline$(\wedge \Sigma . T Y)^{\prime} Y Y$ & $(10 . r \Lambda)^{\varepsilon}$ & YT & سليمة سريريا & $\Lambda$ \\
\hline$(0 \leqslant . \vee q) \backslash Y$ & $(\varepsilon 0, Y)) \cdot \varepsilon$ & Tr. & المجموع & 9 \\
\hline
\end{tabular}

DISCUSSION

المناقشة

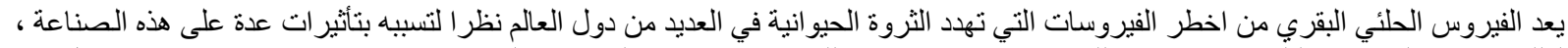

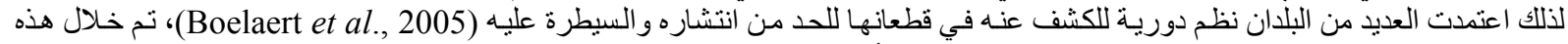

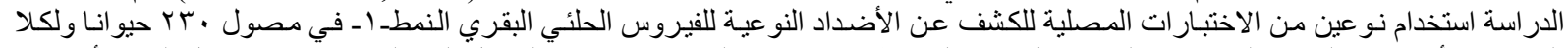

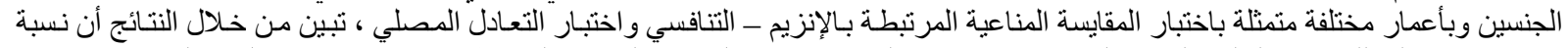

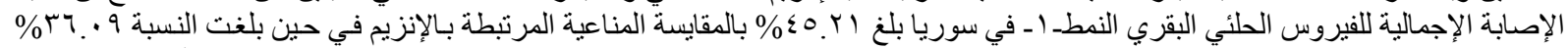

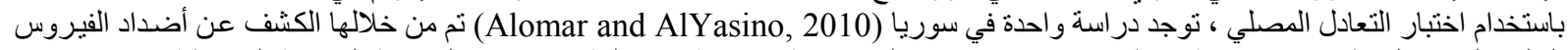

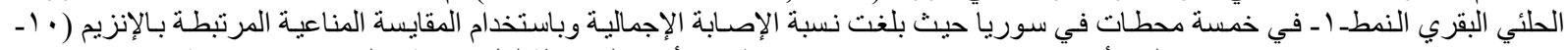

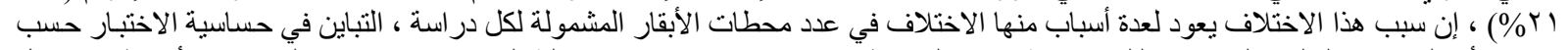

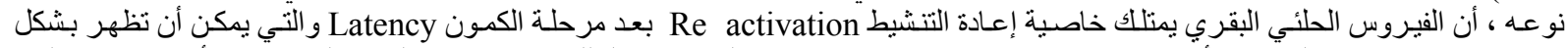

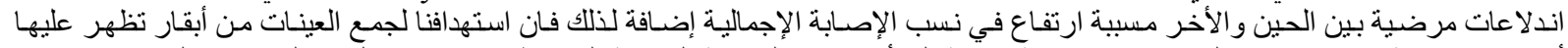

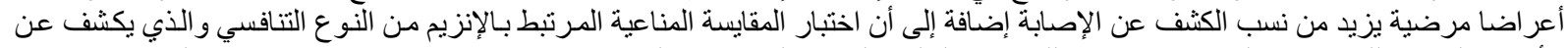

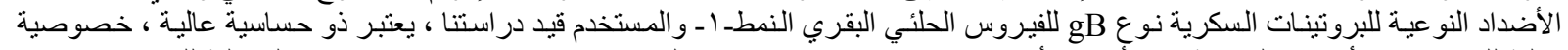

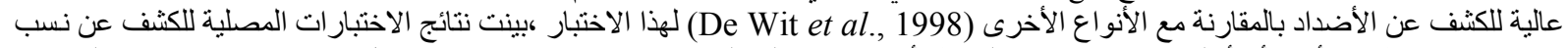

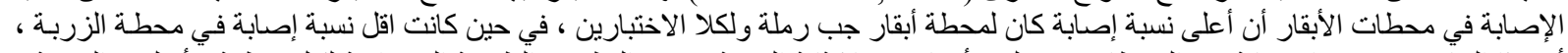

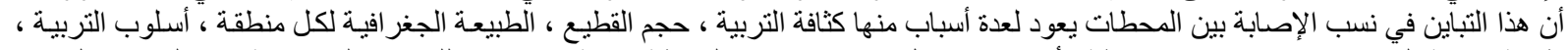

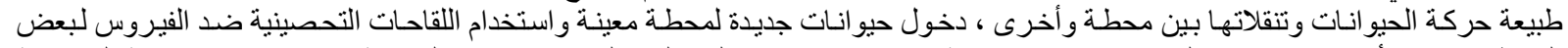

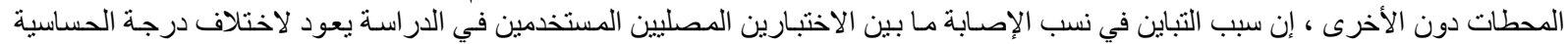

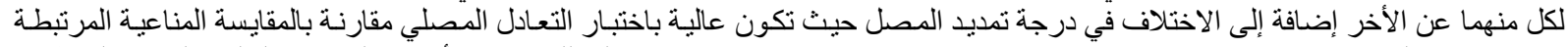

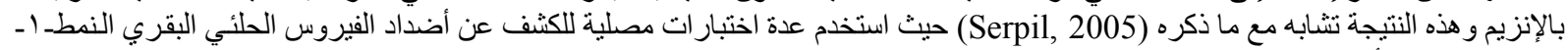

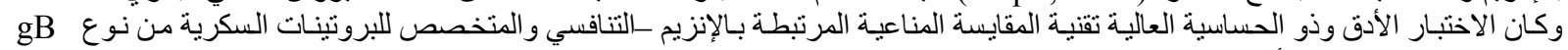

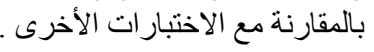

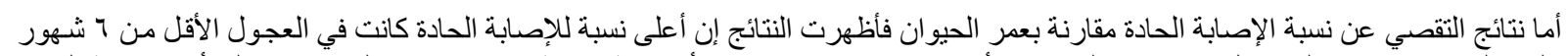

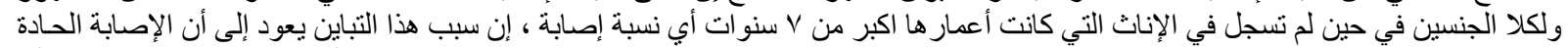

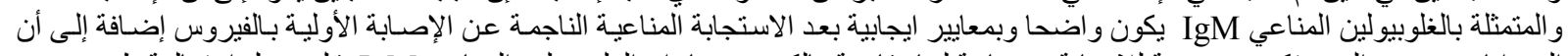

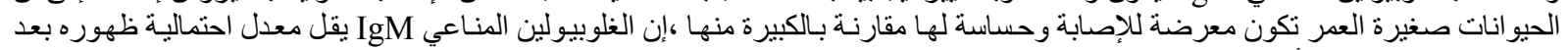

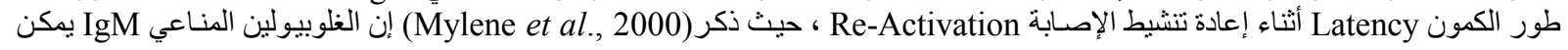




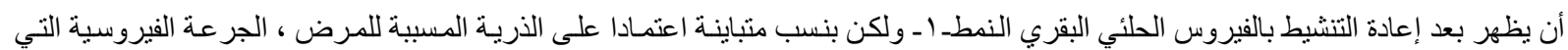

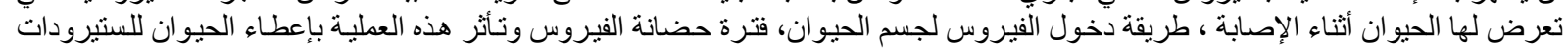

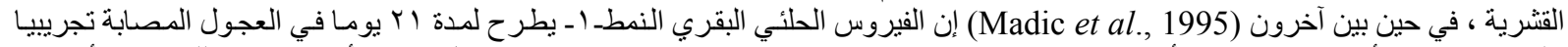

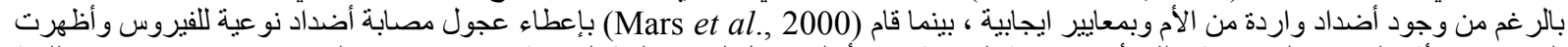

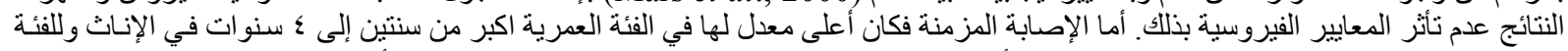

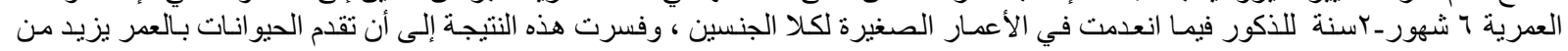

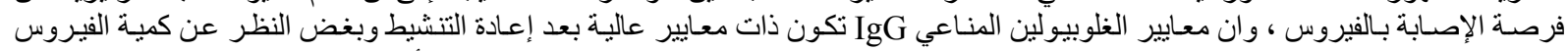

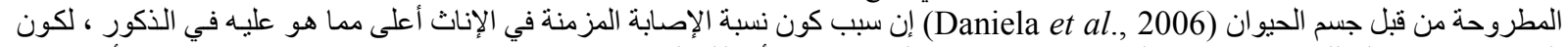

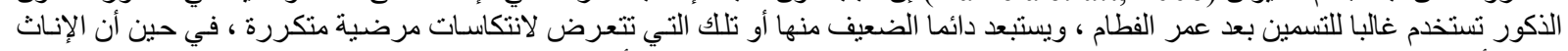

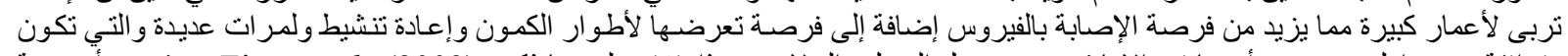

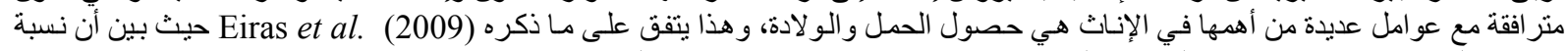

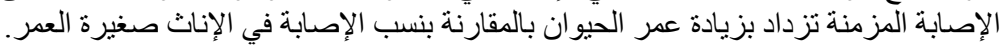

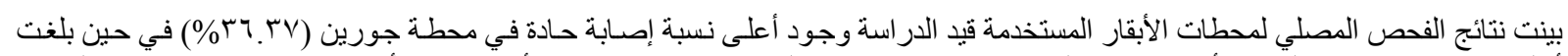

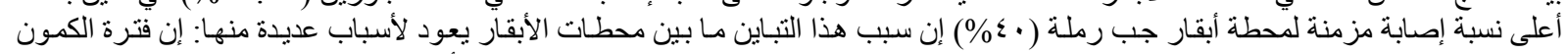

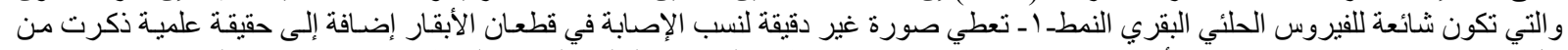

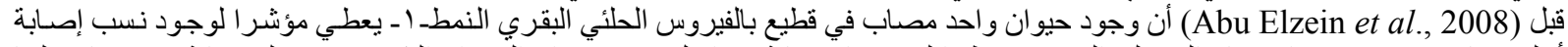

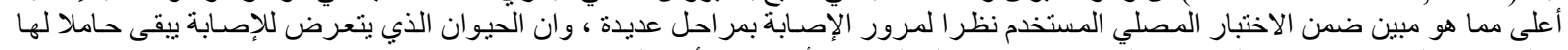
طيلة حياته بالر غم من إعطاء الكثير من الحيو النات نتيجة سالبة لوجود الأضداد في في أمصالها.

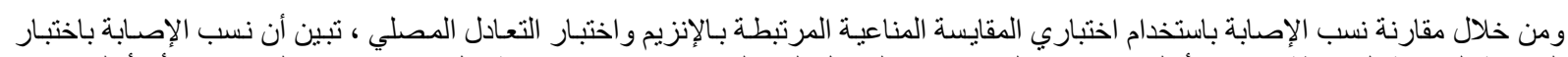

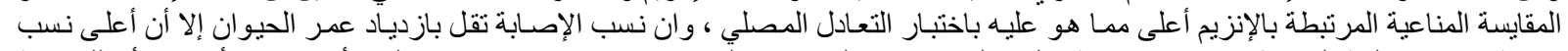

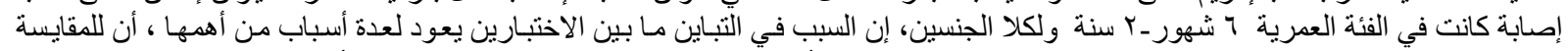

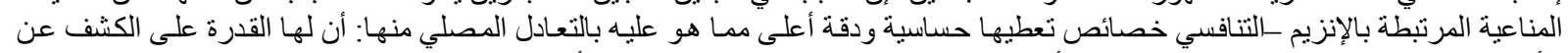

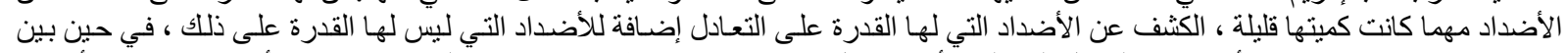

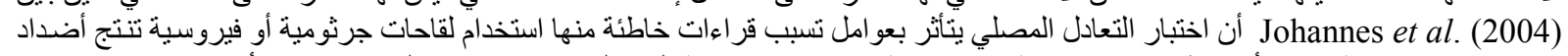

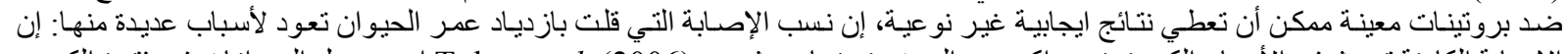

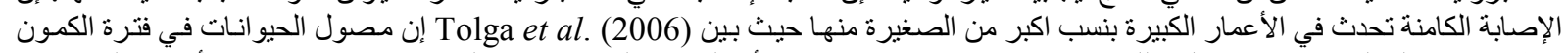

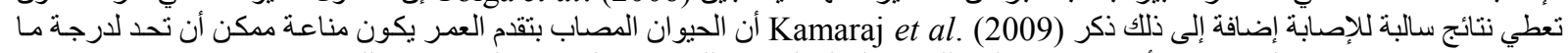

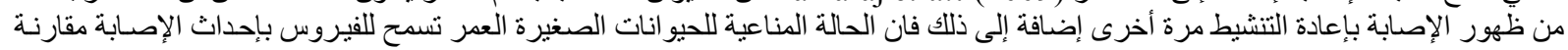
بالحيو انات كبيرة العمر الإصنة.

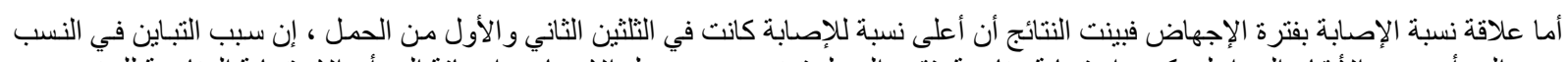

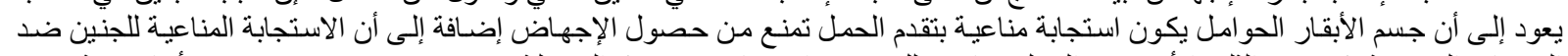

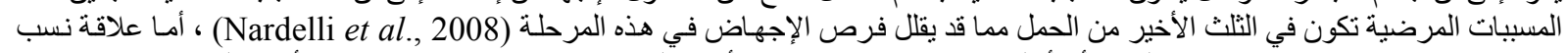

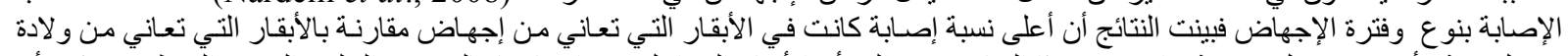

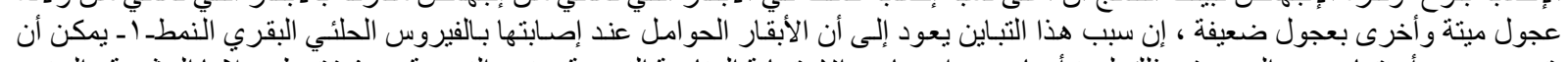

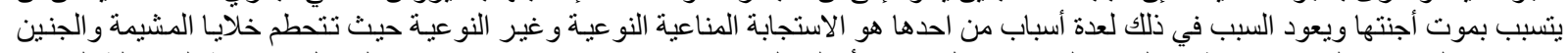

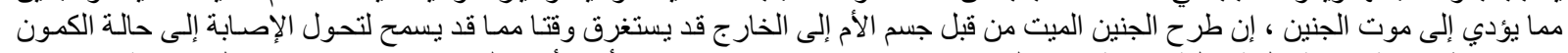

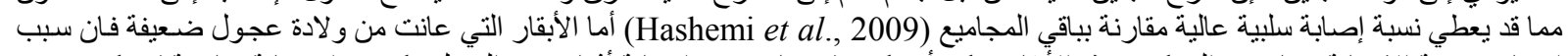

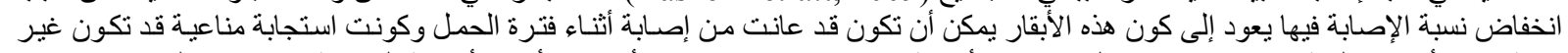

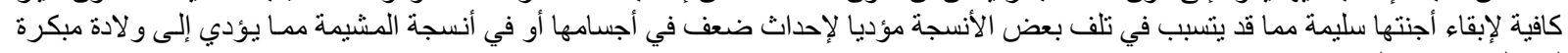

لعجول قد يكون البعض منهاء أنشا مشوها (Winkler et al., 2000).

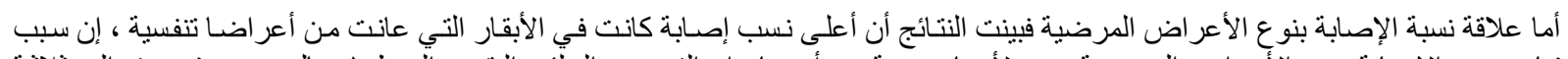

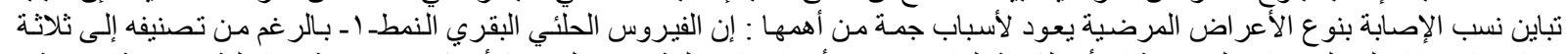

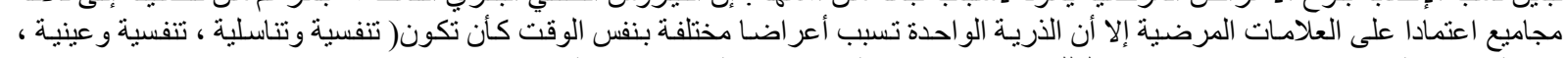

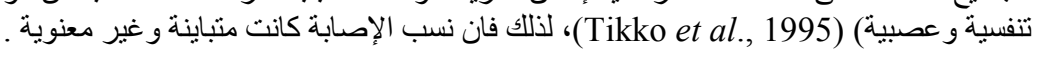

إن انتشار الإصابة بالفيروس الحلئي البقري النمطــ ا ـ في محطات الأبقار في سوريا يعود لأسباب عديدة (مشاهدات حقلية) إن البكاكير يتم تلقيحها

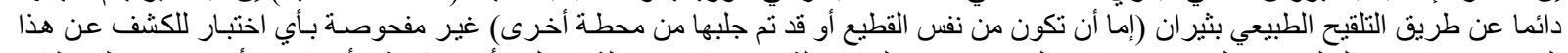

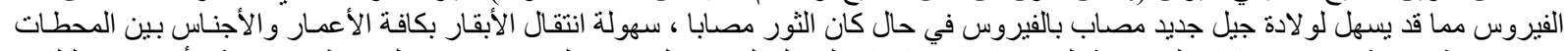

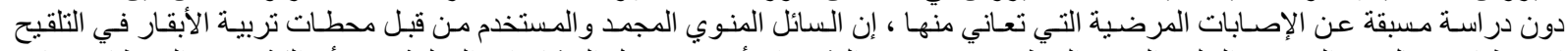

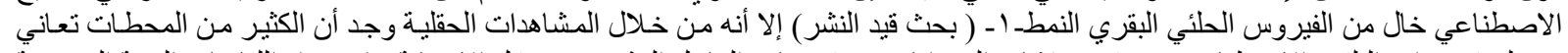

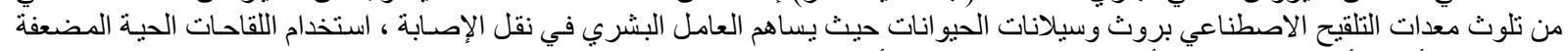

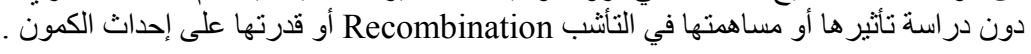

\section{REFERNCES} المصادر

Abu Elzein, E.M.E.; Housawi, F.M.T.; Afaleq, A.I. and Musa, J. (2008): Emergence of clinical infectious bovine rhinotrachitis in Eastern Saudi Arabia. Revue. Elev. Vet. Pays. Trop, 61(1): 11-13.

Alomar, Y. and AlYasino, Y. (2010): Epidemiological study on infectious bovine rhinotrachitis in cattle, International Journal Of Infectious Disease, 14: 32-40. 
Amira, M.E.; Fadol, M.A.; Karrer, A.E. and Elhussin, A.R.M. (2006): IBR virus in Sudan:Epidemiological and Serological studies. Journal of Animal and Veterinary Advances, 5 (12): 1053-1057.

Benoit, M.; Julien, T.; Philippe, K.; Frederic, S. and Etienne, T. (2007): Bovine herpesvirus 1 infection and infectious bovine rhinotrachitis.Vet. Res., 38: 181-209.

Boelaert, F.; Speybroeck, N.; Kruif, A.D.; Aerts, M.; Burzykowski, T.; Molenberghs, G. and Berkvens, D.L. (2005): Risk factor for bovine herpesvirus-1 seropositivity. Preventive Veterinary Medicine, 69: 285295.

Clinton, J. and Shafiqul, C. (2008): A review of the biology of bovine herpesvirus type 1(BHV-1), its role as a cofactor in the bovine respiratory disease complex and development of improved Vaccines. Animal Health Research Reviews, 8(2): 187-205.

Daniela, B.; Ramona, M.; Virgillia, P. and Herman, V. (2006): Evaluation of immune response against infectious bovine rhinotrachitis virus by Immunoenzymatic assay.Buletinul, 63: 1454-1460.

De Wit, J.J.; Hage, J.J.; Brinkhof, J. and Westenbrink, F. (1998): A comparative study of serological tests for use in the bovine herpesvirus 1 eradication programme in the Netherlands. Veterinary Microbiology,61:153-163.

Deka, D.; Ramneek, M.; Maiti, K. and Oberoi, M.S. (2005): Detection of bovine herpesvirus -1 infection in breeding bull semen by virus isolation and polymerase chain reaction. Rev. Sci. Tech. Off. Int. Epiz., 24(3):1085-1094.

Eiras, C.; Dieguez, F.J.; Sanjuan, M.L.; Yus, E. and Aranaiz, I. (2009): Prevalenc of serum antibodies to bovine herpesvirus -1 in cattle in Galicia (NW Spain). Spanish Journal Of Agricutural Research, 7(4): 801-808.

Ezzi, A.; Hatami, A. and Shoukri, M.R. (2011): Study on duration of maternal antibodies in calves against bovine herpesvirus type 1(BHV-1). Archives Of Razi. Institute, 66(1):25-28.

Gonzalez-garcia, M.A.; Arenas-Casas, A.; Carbonero-Martinez, A.; Borge-rodriguez, C.; Garcia-Bocanegra, I.; Maldonado, J.L.; Gomez-Pacheco, J.M. and Perea-Remujo, J.A. (2009): Seroprevalence and risk factors associate with bovine herpesvirus type1(BHV1)infection in non-vaccinated cattle herds in Andalusia (South of Spain). Spanish Journal Of Agricutural Research, 7(3): 550-554.

Hashemi, T.; Rad, G.R.; Naseri, M. and Azizzadeh, M. (2009): Detection of antibody against infectious bovine rhinotrachitis glycoprotein $\mathrm{gE}$ in aborted cattle in Mashhad, Iran. Archives Of Razi Institute, 64(2): 9195.

Johannes, A.K.; Malcolm, B.; Martin, B.; Pierre, K.; Myriam, P.; Gerard, J.W. and Jan, T.V.O. (2004): Evaluation of tests for antibodies against bovine herpesvirus 1 performed in national reference laboratories in Europe. Veterinary Microbiology, 102: 169-181.

Julien, T.; Veronique, K.; Benoit, M.; Francois, M.; Sacha, G.; Alain, V. and Etienne, T. (2006): Ruminant alphaherpesviruses related to bovine herpesvirus 1.Vet. Res., 37: 169-190.

Kamataj, G.; Rana, S.K. and Srinivasan, V.A. (2009): Serological response in cattle immunized with inactivated oil and algel adjuvant vaccines against infectious bovine rhinotrachitis. New Microbiologica, 32: 135141.

Madic, J.; Magdalena, J.; Quak, J. and Oirschot, J.T. (1995): Isotype-specific antibody response to bovine herpesvirus 1 in sera and mucosal secretion of calves after experimental reinfections and after reactivation. Veterinary Immunology and Immunopathology, 47: 81-92.

Maria do Carmo, C.; Edviges Maristela, P.; Ricardo Spacagna, J.; Claudia Pestana, R.; Moacir Marchiori, F. and Helio Jose, M. (2011): Systemic and local antibodies induced by an experimental inactivated vaccine against bovine herpesvirus type 1. Ciencia Rural, 41(2): 307-313.

Mars, M.H.; Rijsewijk, F.A.M.; Maris, M.A.; Van-Orishot, J.T. and Hage, J.J. (2000): Presence of bovine herpesvirus $1 \mathrm{gB}$-serpostive but gE-seronegative Dutch cattle with no apparent virus exposure. veterinary Record, 147(12): 328-331.

Mylene, L.; Vincent, W.; Jacques, G.; Frederic, S.; Gilles, M.; Jean-Jacques, L. and Etienne, T. (2000): Effect of bovine herpesvirus type 1 infection in calves with maternal antibodies on immune response and virus latency. Journal of Clinical Microbiology, 38(5): 1885-1894.

Nardelli, S.; Farina, G.; Lucchini, R.; Valorz, C.; Moresco, A.; Dal Zotto, R. and Costanzi, C. (2008): Dynamic of infection and immunity in a dairy cattle population undergoing an eradication programme for infectious bovine rhinotrachitis (IBR). Preventive Veterinary Medicine, 85: 68-80.

Rai, A. (2005): Methods in veterinary virology, Indian Veterinary research Institute, India, PP:50-52.

Serpil, Y. (2005): Acomparative evaluation of bovine herpesvirus -1 infection by Enzyme Linked Immunosorbant assay and serum Neutralization test in Konya Province. Hayvancihk Arastrima Dergisi, 15: $5-8$.

Tikko, S.K.; Campos, M. and Babiuk, L.A. (1995): Bovine herpes virus 1 (BHV1): Biology, Pathogenesis, and Control. Adv. Virus. Res., 45: 191-223.

Tolga, M.T.; Yakup, Y.; Nural, E. and Burak, A.G. (2006): The prevalence of bovine herpesvirus type 1(BHV1) and bovine Leukemia virus (BLV) in selected dairy cattle herds in Aydin Province, Turkey. Turk Journal Veterinary Animal Science, 30: 353-357.

Winkler, M.T.; Doster, A. and Jones, C. (2000): Persistence and reactivation of bovine herpesvirus 1 in the tonsils of latency infected calves. Journal Of Virology, 74(11): 5337-5346. 